\title{
Boundary Layer Heat Transport in turbulent Rayleigh-Bénard Convection in Air
}

\author{
Ronald du Puits ${ }^{1, *}$, Christian Resagk ${ }^{1}$, and Christian Willert ${ }^{2}$ \\ ${ }^{1}$ Technische Universität Ilmenau, POB 100565, 98684 Ilmenau \\ ${ }^{2}$ German Aerospace Center, 51170 Cologne
}

\begin{abstract}
We study the convective heat transfer in a large-scale Rayleigh-Bénard (RB) experiment which is called the "Barrel of Ilmenau". We present the results of flow visualization and Particle Image Velocimetry (PIV) measurements of the near wall flow field in a plane perpendicular to the surface of the heated bottom plate. The experiment was run in a smaller rectangular inset that was placed inside the larger barrel. The Rayleigh number amounts to $R a=1.4 \times 10^{10}$. The aspect ratios were $\Gamma_{x}=1$ in flow direction and $\Gamma_{y}=0.26$ perpendicular to the vertical flow plane. The measurements have been undertaken using a $2 \mathrm{~W}$ continuous wave Laser in combination with a light sheet optics and various cameras. Due to the slender geometry of the cell, the mean wind is confined in one direction where the Laser light sheet is aligned parallel. The flow was seeded with droplets of $1 \ldots 2 \mu \mathrm{m}$ size generated using an ordinary fog machine. Flow visualization as well as the PIV data clearly show the intermittent character of the boundary layer flow field that permanently switches between "laminar" and "turbulent" phases.
\end{abstract}

(c) 2014 Wiley-VCH Verlag GmbH \& Co. KGaA, Weinheim

\section{Introduction}

Many natural or technical flows are associated with a heat transfer from a hot or cold solid surface to a surrounding fluid. However, particularly in case of highly turbulent flows the knowledge about the temperature and the velocity field inside the convective boundary layer, and thus, the predictability of the local heat transfer coefficient is still limited. We study this process in a large-scale Rayleigh-Bénard (RB) experiment which is called the "Barrel of Ilmenau" (see Figure 1, for more details see [1]). It meets two important criteria, a very high Rayleigh number of $R a_{\max }=10^{12}-R a=\left(\beta g \Delta T H^{3}\right) /(\nu \kappa)$, with $\beta$ - thermal expansion coefficient, $g$ - gravitational acceleration, $\Delta T$ - temperature difference, $H-$ thickness of the fluid layer, $\nu$ - kinematic viscosity, $\kappa$ - thermal diffusivity - and a large size of $7.15 \mathrm{~m}$ in diameter and $6.30 \mathrm{~m}$ in height. The particular advantage of this extra-large facility is the fact that the boundary layer is of the order of tens of millimeters (depending on $\mathrm{Ra}$ ) which permits a maximum of spatial resolution for all measurements compared to any other RB facility in the world.

* Corresponding author: e-mail ronald.dupuits@tu-ilmenau.de, phone +493677 691353, fax +493677693683

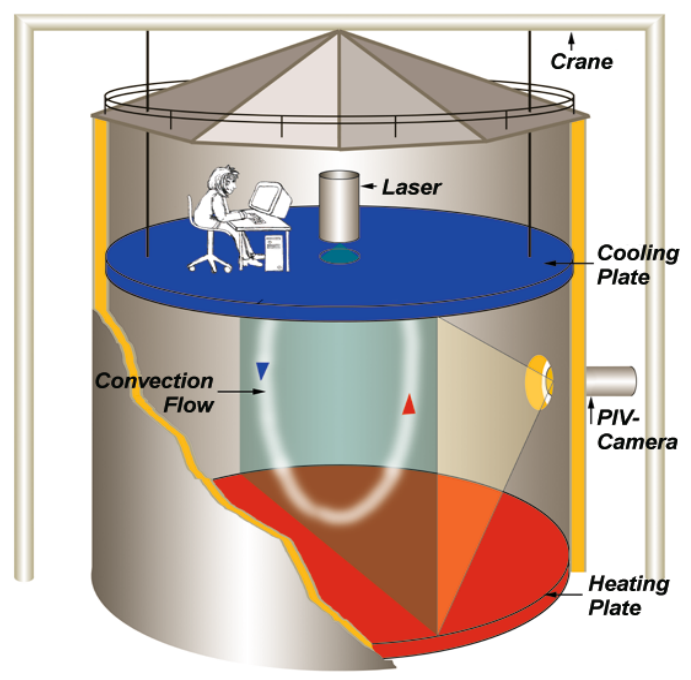

Fig. 1: Sketch of the large-scale RB experiment "Barrel of Ilmenau".

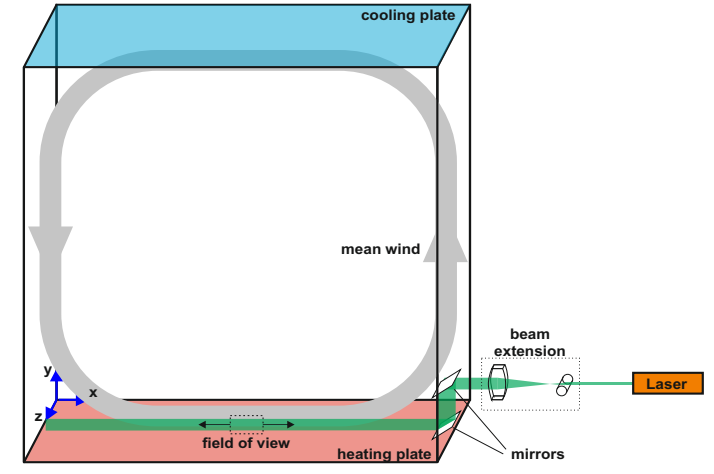

Fig. 2: Set-up of the flow visualization at the rectangular RB cell inside the large-scale facility. 


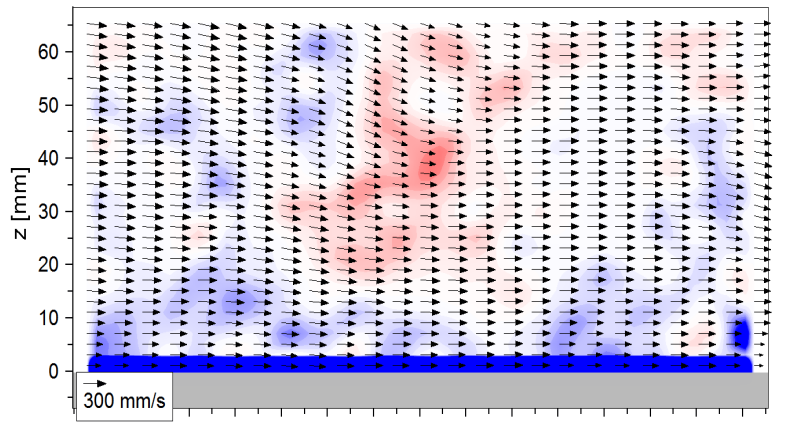

a)

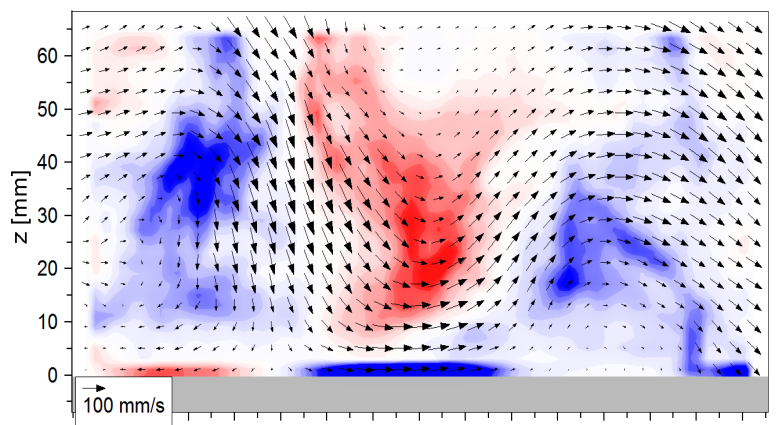

c)
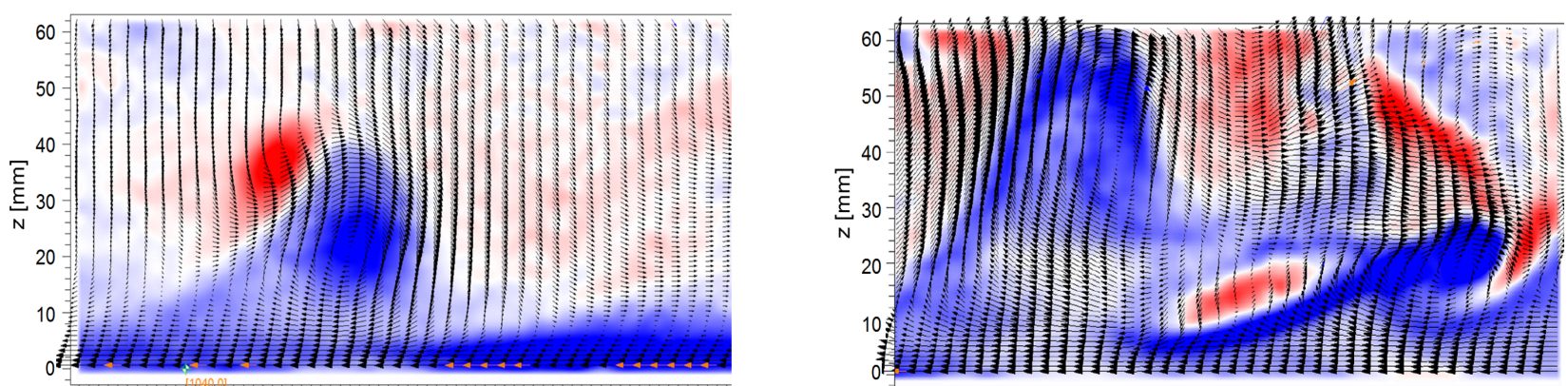

b)

d)

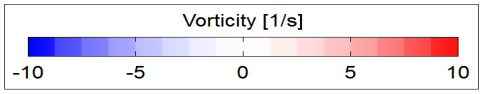

Fig. 3: Snapshots of the characteristic flow phases: laminar flow phase (a), transitional flow phase due to boundary layer instabilities (b), transitional flow phase due to the entrainment of turbulent kinetic energy from the bulk (c), and fully turbulent flow phase (d).

\section{Results}

We present the results of flow visualization and Particle Image Velocimetry (PIV) measurements of the near wall flow field in a plane perpendicular to the surface of the heated bottom plate. The experiment was run in a smaller rectangular inset that was placed inside the larger barrel (see Figure 2). The Rayleigh number amounts to $R a=1.4 \times 10^{10}$. The aspect ratios were $\Gamma_{x}=1$ in flow direction and $\Gamma_{y}=0.26$ perpendicular to the vertical flow plane. The measurements have been undertaken using a $2 \mathrm{~W}$ continuous wave Laser in combination with a light sheet optics and various cameras. Due to the slender geometry of the cell, the mean wind is confined in one direction where the Laser light sheet is aligned parallel. The flow was seeded with droplets of $1 \ldots 2 \mu \mathrm{m}$ size generated using an ordinary fog machine.

Flow visualization as well as the PIV data clearly show the intermittent character of the boundary layer flow field that permanently switches between various flow regimes [2,3]. Four characteristic flow phases could be identified: i) a laminar flow phase, ii) a transitional flow phase due to boundary layer instabilities, iii) a transitional flow phase due to the entrainment of turbulent kinetic energy from the bulk, and iv) a fully turbulent flow phase (see Figure $3 \mathrm{a}-\mathrm{d}$ ). For the first time, it could be demonstrated that the entrainment of turbulent structures from the mean wind into the boundary layer acts, besides the destabilization due to its inner shear as a second mechanism on its path towards turbulence. Both contributions must be considered if predicting the critical bound towards the "ultimate regime" of thermal convection [4].

Acknowledgements We wish to acknowledge the Deutsche Forschungsgemeinschaft under the grant number PU 436/1-1 and PU 436/1-2, the European Union under the COST program MP0806 as well as the Thueringer Ministerium fuer Wissenschaft, Forschung und Kunst for the financial support of the work reported here.

\section{References}

[1] R. du Puits, C. Resagk, and A. Thess, New J. Phys. 15, 013040 (2013).

[2] R. du Puits, J. Rilk, C. Resagk, and A. Thess, arXiv:1209.6201v1 [physics.flu-dyn].

[3] R. du Puits, L. Li, C. Resagk, and A. Thess, C. Willert, Phys. Rev. Lett., to be published.

[4] R.-H. Kraichnan, Phys. Fluids 5, 1374(1962). 